\title{
Deterministic and Stochastic Becker-Döring equations: Past and Recent Mathematical Developments
}

\author{
E. Hingant R. Yvinec
}

August 31, 2016

\section{Contents}

1 Introduction 1

2 Deterministic mean-field theory 2

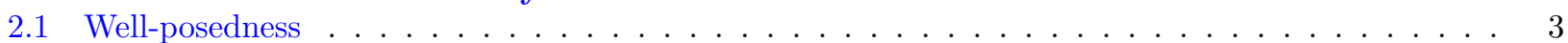

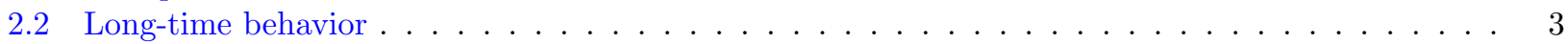

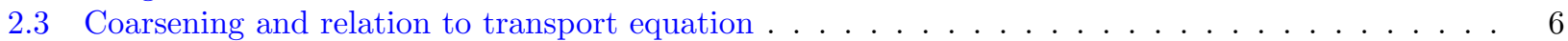

2.4 Time-dependent properties, Metastability and Classical nucleation theory . . . . . . . . . . 10

3 Stochastic Becker-Döring model 11

3.1 Definition and State-space . . . . . . . . . . . . . . . . . . . . . . . . 11

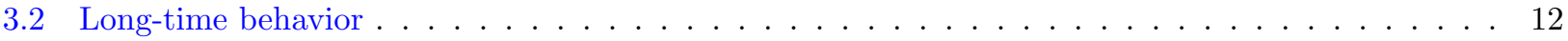

3.3 Large Number and relation to deterministic Becker-Döring . . . . . . . . . . . . . . . . 13

3.4 Time-dependent properties, Metastability and stochastic nucleation theory . . . . . . . . . . 14

$\begin{array}{ll}\text { Acknowledgment } & 16\end{array}$

$\begin{array}{lc}\text { References } & 16\end{array}$

\section{Introduction}

The Becker-Döring (BD) equations goes back to the seminal work "Kinetic treatment of nucleation in supersaturated vapors" by Becker and Döring (1935), which gave rise to the name of the model. Later on, Burton (1977) popularized the use of such equations to study condensations phenomena at different pressures. Since then, applications of this model range from physics, chemistry to biology. Recently, the book edited by Schmelzer (2005) make an inventory of several applications of nucleation and phase transition theory. Let us also point out recent applications of Becker-Döring or related coagulation-fragmentation models in biology, specifically to protein aggregation in neurodegenerative diseases e.g. the works by Linse and Linse (2011), Prigent et al. (2012), Alvarez-Martinez et al. (2011), Budrikis et al. (2014), Eden et al. (2015), Davis and Sindi (2016), Eugene et al. (2016) and Doumic et al. (2016), also Hu and Othmer (2011) worked on polymerization of actin filaments, Hoze and Holcman (2015, 2014) on assembly of virus capsids, Bressloff (2016) on vesicular transport, and Hoze and Holcman (2012) for telomere clustering.

In its survey, Slemrod (2000) said the BD equations "provide perhaps the simplest kinetic model to describe a number of issues in the dynamics of phase transitions". This is maybe one of the reason these equations received lot of attentions from many mathematicians. But being simple these equations do not prevent its richness and difficulties. Our intention here, is: on one hand, to complete the review by Slemrod with new results; and on the other hand, to give a parallel with the stochastic version of these equations, which reveals a lot of new interesting problems. We also mention the review by Wattis (2006) which contains many qualitative and exact properties of the solutions in the deterministic context, and the pedagogical notes by Penrose (2001). But, few stochastic review of the BD model is available, we can only mention the seminal work by Aldous (1999) which treats the so-called Smolukowsky coagulation equations.

The model consists in describing the repartition of clusters by their size $i \geq 1$, i.e. the number of particles that composed them. Clusters belong to a "solvent" in much smaller proportion and are assumed to be spatially homogeneously distributed. Along their motion, clusters give rise to two types of reactions, namely the BeckerDöring rules: 
1. A cluster of size 1 , commonly called monomer or elementary particle, may encounter a cluster of size $i \geq 1$ to coalesce and give rise to a cluster of size $i+1$.

2. A cluster of size $i \geq 2$ may release spontaneously a monomer resulting in a cluster of size $i-1$.

These can be summarized by the set of kinetic reactions, for each $i \geq 1$,

$$
C_{1}+C_{i} \underset{b_{i+1}}{\stackrel{a_{i}}{\rightleftharpoons}} C_{i+1}
$$

where $C_{i}$ denotes clusters consisting of $i$ particles. Coefficients $a_{i}$ and $b_{i+1}$ stand, respectively, for the rate of aggregation and fragmentation. These may depend on the size of clusters involved in the reactions and typical coefficients are derived by Penrose (1997) and Niethammer (2003):

$$
a_{i}=i^{\alpha}, \quad b_{i+1}=a_{i+1}\left(z_{s}+\frac{q}{(i+1)^{\gamma}}\right), \quad i \geq 1 .
$$

for $0 \leq \alpha<1, z_{s}>0, q>0$ and $0<\gamma<1$. This choice is in agreement with original derivation where $a_{i} \approx i^{2 / 3}$, $b_{i} \approx a_{i} \exp \left(G i^{-1 / 3}\right)$. In particular, the diffusion-limited case of monomers clustering into sphere corresponds to $\alpha=1 / 3, \gamma=1 / 3$ in $3 \mathrm{D}$ and to $\alpha=0, \gamma=1 / 2$ in 2D, while the interface-reaction-limited case corresponds to $\alpha=2 / 3, \gamma=1 / 3$ in $3 \mathrm{D}$ and $\alpha=1 / 2, \gamma=1 / 2$ in 2D. We refer also to Penrose and Buhagiar (1983) for a method on deriving coefficients. Note that all along the survey we assume the natural hypothesis $a_{i}$ and $b_{i+1}$ are non-negative for all $i \geq 1$, without referring to this again.

In its mean-field version, or deterministic, the BD model is an infinite set of ordinary differential equations for the time evolution of each concentrations (numbers per unit of volume) of clusters made of $i$ particles. In its stochastic version, the BD model is a continuous time Markov chain, on a finite state space. We divide the remainder of this survey in two parts for the respective versions.

\section{Deterministic mean-field theory}

The general formulation of the deterministic Becker-Döring equations, as studied today, seems to go back to Burton (1977) and was popularized among mathematicians by Penrose and Lebowitz (1979) (indeed, the equations studied in the original work by Becker and Döring (1935) slightly differ, see comment later on). It assumes the system behaves homogeneously in space with a high number of clusters, and considers concentrations $c_{i}(t)$ (unit per volume) of clusters with size $i \geq 1$ at time $t \geq 0$. It deals with classical law of chemistry (Law of Mass Action), the coagulation is considered as a second order reaction while the fragmentation is a first-order (linear) reaction. The flux associated to the kinetic scheme (1) is thus given, for each $i \geq 1$, by

$$
J_{i}=a_{i} c_{1} c_{i}-b_{i+1} c_{i+1}
$$

Considering all the fluxes involved in the variations of the concentration of each $c_{i}$ entails the infinite system of differential equations, namely the Becker-Döring equations:

$$
\begin{aligned}
\frac{d}{d t} c_{1} & =-J_{1}-\sum_{i \geq 1} J_{i}, \\
\frac{d}{d t} c_{i} & =J_{i-1}-J_{i},
\end{aligned}
$$

for every $i \geq 2$. The system considered here has no source nor sink. Consequently, for the total amount of monomers, we should have, for all $t \geq 0$,

$$
\sum_{i \geq 1} i c_{i}(t)=\rho
$$

where $\rho$ is a constant, called through the survey: mass of the system. Formal computations on the solution of the system (4-5), interverting infinite sum, lead to this statement. Remark, the constant $\rho$ is entirely determined by the initial condition given at time $t=0$. In this section we try to expound the main theory around these equations. In particular, we exclude many variants such as the original constant monomer formulation, which is then an infinite linear system (e.g. Penrose, 1989, Kreer, 1993 or King and Wattis, 2002), the finite-dimensional truncated system (e.g. Duncan and Soheili, 2001 or Duncan and Dunwell, 2002), generalization such as micelles formation (e.g. Coveney and Wattis, 1996) or including space with cluster diffusion (e.g. Laurençot and Wrzosek, 1998) or lattice models (e.g. Penrose and Buhagiar, 1983).

We separated this section between well-posedness, long-time behavior, scaling limit, and some time-dependent properties. 


\subsection{Well-posedness}

The first general result on existence and uniqueness on Becker-Döring equations is due to Ball et al. (1986) which really start the mathematical analysis of BD equations. The authors state many of the fundamental properties of the solutions belonging to the Banach space

$$
X^{+}:=\left\{x \subset \mathbb{R}_{+}^{\mathbb{N}}: \sum_{i \geq 1} i x_{i}<+\infty\right\},
$$

which arises naturally in view of the balance of mass Eq. (6). We recall first the notion of solutions to BD equations.

Definition 1. Let $T \in(0,+\infty]$ and $c^{\text {in }} \in X^{+}$. A solution to the Becker-Döring equations $(4-5)$ on $[0, T)$ with initial data $c^{\text {in }}$, is a function $c:[0, T) \rightarrow X^{+}$which writes $c:=\left(c_{i}\right)_{i \geq 1}$ and such that: $\sup _{t \in[0, T)}\|c(t)\|_{X}<+\infty$; for all $t \in[0, T)$, we have $\sum_{i>1} a_{i} c_{i} \in L^{1}(0, t)$ and $\sum_{i \geq 2} b_{i} c_{i} \in L^{1}(0, t)$; Eqs. (4-5) hold almost every $t \in[0, T)$ and $c(0)=c^{\text {in }}$.

One of the fundamental fact, proved by Ball et al. (1986), is that any solution to the BD equations satisfies the balance of mass Eq. (6) at all finite time (Corolary 2.6). In particular, any solution to the BD equations avoids the so-called gelation phenomenon (in finite time) which can occur in general coagulation-fragmentation equations (e.g. Escobedo et al. 2003). Ball et al. (1986) also proved propagation of moments (Theorem 2.2) and regularity properties of the solutions (Theorem 3.2). Finally, they state a general existence result for sublinear coagulation rate and uniqueness with an extra-moment on the initial condition (see below Theorem 1). In short, the work by Ball et al. (1986) covered the essential properties of BD equations, build the foundations for the analysis of BD equations, and should be a companion for whom want to work with.

We go back to well-posedness, for which Laurençot and Mischler (2002) complement the result, by Ball et al. (1986), proving the uniqueness without extra condition on the initial data but assuming a growth condition on the fragmentation rate, viz. there exists a constant $K>0$ such that, for every $i \geq 2$,

$$
a_{i}-a_{i-1} \leq K, \quad b_{i}-b_{i+1} \leq K .
$$

We summarize these results in the following theorem.

Theorem 1 (Well-posedness, Ball et al. (1986), Laurençot and Mischler (2002)). Let $c^{\text {in }} \in X^{+}$. Assume alternatively either a) $a_{i}=O(i)$ and $\sum_{i \geq 1} i^{2} c_{i}^{\text {in }}<+\infty$, or b) the growth condition (7). The Becker-Döring equations (4-5) have a unique solution $c$ on $[0,+\infty)$ associated with the initial data $c^{\text {in }}$. Moreover, for all $t \geq 0$,

$$
\sum_{i \geq 1} i c_{i}(t)=\sum_{i \geq 1} i c_{i}^{\text {in }}
$$

In fact the uniqueness by Ball et al. (1986) is slightly more subtle, see their Theorem 3.6. Also, they proved that $a_{i}=O(i)$ is almost optimal. Indeed, their Theorem 2.7 states: if $\lim _{i \rightarrow \infty} a_{i} / i=+\infty$ and $\lim _{i \rightarrow \infty} b_{i+1} / a_{i}<$ $+\infty$, then for some initial condition (with relatively fat tail) still belonging to $X^{+}$the BD system has no-solution. This suggests that, for super-linear coagulation rate, we cannot hope existence for a large class of initial data without a sufficient control on the fragmentation rate. Since mass is preserved, fragmentation should balance the formation of "big" clusters. It seems very few results exist for such class of coefficients, except Wattis et al. (2004) who considered exponential coefficients.

Finally, we mention that a proof of existence to the BD equations is self-contained in the nice proof by Laurençot (2002) for a more general model (discrete coagulation with multiple fragmentation). It relies, as for the proof given by Ball et al. (1986), on a truncated system up to a size $N$ and compactness arguments to obtain the limit $N \rightarrow+\infty$. But here Laurençot (2002) took advantage of the propagation of super-linear moments and a De La Vallé Poussin lemma to prove compactness.

\subsection{Long-time behavior}

The long-time behavior of the BD system brings some of its most interesting properties, and we will see this is still under active research. Through this section we will always assume that both $a_{i}$ and $b_{i+1}$ are positive for each $i \geq 1$. This avoids many pathological cases, in some sense, if one of them cancel it "breaks the communication" between clusters in one side or another. Nonetheless, we mention the interesting cases (not detailed here) where either $a_{i}=0$ or $b_{i+1}=0$, for every $i \geq 1$, which have been treated again by Ball et al. (1986)! We start with a subsection which deals with convergence to equilibrium. Then, we will see the most recent results on the exponential stability of the equilibrium. 


\subsubsection{Convergence to equilibrium}

The equilibrium candidates, at plural, of the BD equations are obtained by canceling the fluxes $J_{i}$, for each $i \geq 1$, as defined in Eq. (3). After straightforward manipulation of the fluxes, the candidates form a one-parameter family, indexed by a variable $z \geq 0$, and are given by the expressions

$$
\bar{c}_{i}(z)=Q_{i} z^{i}, \quad \text { where } \quad Q_{i}=\frac{a_{1} a_{2} \cdots a_{i-1}}{b_{2} b_{3} \cdots b_{i}},
$$

for every $i \geq 1$, with the convention $Q_{1}=1$. For example, the case related to Eq. (2) gives (e.g. Niethammer, 2003), for large $i$,

$$
Q_{i} \approx \frac{C}{i^{\alpha} z_{s}^{(i-1)}} \exp \left(-\frac{q}{(1-\gamma) z_{s}} i^{1-\gamma}\left(1+O\left(i^{-\gamma}\right)\right)\right) .
$$

To find the right equilibrium, which reduces to find the value of $\bar{c}_{1}=z$, one should use the balance of mass Eq. (6) which we know to be satisfied at any finite time. Hence, this leads us to consider the power series, given by the mass of the equilibrium candidates,

$$
\sum_{i \geq 1} i Q_{i} z^{i}
$$

which radius of convergence is denoted by $z_{s}$. Such radius is obtained from the rates functions since the CauchyHadamard theorem says $1 / z_{s}:=\lim \sup _{i \rightarrow \infty} Q_{i}^{1 / i}$. This becomes the heart of the existence of a critical mass in $\mathrm{BD}$ equations since the values taken by the series may not define a bijection from $\left[0, z_{s}\right)$ into $[0,+\infty)$. Indeed, set $\rho_{s}$ be the upper value taken by the series on $\left\{z<z_{s}\right\}$. It can occur that $\rho_{s}$ is finite, in which case we already know that there is no equilibrium candidate with mass $\rho>\rho_{s}$. Hence, this leads to a dichotomy in the long-time behavior of the BD equations whether or not the mass of the solution considered is less than $\rho_{s}$, named the critical mass. We may refer to sub-critical solution when the mass $\rho<\rho_{s}$, critical solution when $\rho=\rho_{s}$ and super-critical solution when $\rho>\rho_{s}$.

The Becker-Döring equations are part of the kinetic equations. These later have a long story, led by the celebrated Boltzmann equations, which are of course completely out of the scope of this paper, maybe the reader could refer to Cercignani (1990). The key concept in these equations is the entropy (sometimes called energy) which, in mathematical words, is a Lyapounov functional and governs the trend to equilibrium. Namely, the entropy arising in $\mathrm{BD}$ equations is given by the expression

$$
H(c)=\sum_{i \geq 1} c_{i}\left(\ln \left(\frac{c_{i}}{Q_{i}}\right)-1\right) .
$$

This is because, formally, the $H$ decreases along the solutions $c$ (and is bounded from below), as

$$
\frac{d}{d t} H(c(t))=-D(c(t))
$$

where the dissipation is

$$
D(c):=-\sum_{i \geq 1}\left(a_{i} c_{1} c_{i}-b_{i+1} c_{i+1}\right)\left(\ln \left(a_{i} c_{1} c_{i}\right)-\ln \left(b_{i+1} c_{i+1}\right)\right) .
$$

Remark, since ln is increasing, the dissipation $D$ is non negative. Depending on the properties you are looking for, this is possible to define the relative entropy functional, with same dissipation term, and given by the expression

$$
H\left(c \mid c^{\rho}\right)=\sum_{i \geq 1} c_{i}\left(\ln \left(\frac{c_{i}}{c_{i}^{\rho}}\right)-1\right)+\sum_{i \geq 1} c_{i}^{\rho},
$$

where $c^{\rho}$ is the equilibrium candidate, with mass $\rho$, i.e. the components are given by Eq. (8) for which $z$ is chosen such that $\sum_{i \geq 1} i Q_{i} z^{i}=\rho$. The second term in the right-hand side, ensuring non-negativity, is some times omitted. Hence, in the case initially $H\left(c^{\mathrm{in}} \mid c^{\rho}\right)<+\infty$, we should have $D(c(t)) \rightarrow 0$ as $t \rightarrow \infty$ as we can see in

$$
0 \leq H\left(c(t) \mid c^{\rho}\right)+\int_{0}^{t} D(c(s)) d s \leq H\left(c^{\mathrm{in}} \mid c^{\rho}\right) .
$$

And remarking that $D=0$ corresponds, see its definition, to $J_{i}=0$ for all $i \geq 1$, we have a good reason to go ahead with the functional $H$. The hard work is to prove rigorous properties on the entropy and relative entropy, along the solutions. Again Ball et al. (1986) set the basic. The authors give many results, among others, continuity properties of the entropy functional (Proposition 4.5) and minimizing sequence properties (Theorem 4.4). Also, they proved the key ingredient that Eq. (10) holds for a large class of rate (Theorem 4.8). Finally, in their Theorem 4.7, they proved the so-called $H$-theorem (by analogy with the celebrating Boltzmann $H$-theorem), which is a rigorous justification of (9). 
Theorem 2 (H-theorem, Ball et al. (1986)). Assume $z_{s}>0, \liminf _{i \rightarrow \infty} Q_{i}^{1 / i}>0, a_{i}=O(i / \ln i)$ and $b_{i}=$ $O(i / \ln i)$. If $c$ is a solution to the Becker-Döring equations (4-5) on $[0, T)$, for some $T \in(0,+\infty]$, with initial condition $c^{\text {in }} \neq 0$ belonging to $X^{+}$, then dissipation of entropy Eq. (9) holds almost every $t \in[0, T)$.

Note that linear growth $a_{i}, b_{i} \sim i$ is not allowed. Fewer assumptions on the rate of fragmentation is possible, adapting the results obtained for general coagulation-fragmentation equation by Carr and da Costa (1994) and later by Cañizo (2007). Now we state the main asymptotic results. A very general result in the case $z_{s}=+\infty$ is available from Theorem 5.4 by Ball et al. (1986). But the more interesting case is $0<z_{s}<+\infty$ for which a dichotomy occurs. This is treated for particular initial conditions and rates by Ball et al. (1986), and then extended to general initial conditions in Ball and Carr (1988). Finally, it was refined by Slemrod (1989) for a class of rates allowing linear growth, see its Theorem 5.11, which we state below.

Theorem 3 (Convergence to equilibrium, Slemrod (1989)). Let $c^{\text {in }} \in X^{+}$with mass $\rho$ and such that $H\left(c^{\text {in }}\right)<$ $+\infty$. Assume $a_{i}=O(i), b_{i}=O(i)$ and $\lim _{i \rightarrow+\infty} Q_{i}^{1 / i}=1 / z_{s}$ exists $\left(z_{s}>0\right)$. Assume moreover there exists $z \in\left[0, z_{s}\right]$ such that $a_{i} z \leq b_{i}$ for sufficiently large $i$. Finally, let $c$ be the unique solution to the Becker-Döring equations $(4-5)$ on $[0,+\infty)$ with initial data $c^{\text {in }}$. We have:

(a) If $0 \leq \rho \leq \rho_{s}$, then $\lim _{t \rightarrow+\infty} \sum_{i \geq 1} i\left|c_{i}(t)-c_{i}^{\rho}\right|=0$.

(b) If $\rho>\rho_{s}$, then, for every $i \geq 1, \lim _{t \rightarrow+\infty} c_{i}(t)=c_{i}^{\rho_{s}}$.

In both case we recall that $c^{\rho}$ is the equilibrium given by Eq. (8) with mass $\rho$.

Surprisingly, in point (b), while the solution has mass $\rho$ for all times, as the time goes to infinity, it converges in a weak sense (component by component) to a solution having a strictly inferior mass. In this theory, the difference $\rho-\rho_{s}$ is interpreted as the formation of particles with infinite size and of different nature, phenomenon called phase transition. In Section 2.3.1 we will describe in more details this phenomenon.

The proof consists first in proving that the $w\left(c^{\text {in }}\right)$ - limit set consist of equilibrium candidate $c^{\rho^{\prime}}$ with mass $\rho^{\prime}$ less than $\min \left(\rho, \rho_{s}\right)$. This is achieved by compactness of the orbit, analyzing the time-translation, and by regularity of the $c_{1}$ which requires in particular $b_{i}=O(i)$ (see Theorem 3.2 in Ball et al. (1986)), contrary to the known existence result stated above in Theorem 1 . Then, the limit is selected thanks to the dissipation (10). A key ingredient is the continuity property of $c \mapsto H\left(c \mid c^{\rho}\right)$ which holds if and only if $\lim _{i \rightarrow+\infty} Q_{i}^{1 / i}$ exists and $\rho=\rho_{s}$, see Proposition 4.5 by Ball et al. (1986). Note, the condition $a_{i} z \leq b_{i}$ comes from the Theorem 2 by Ball and Carr (1988), and re-used by Slemrod (1989), which ensures the tail of the $c_{i}$ 's decays sufficiently fast (fragmentation dominates). We point out that these two last conditions are needed to select the right equilibrium, while convergence to some equilibrium is "always" satisfied, see Theorem 5.10 by Slemrod (1989).

We finish by a comment on the case where $z_{s}=0$, corresponding to a strong coagulation rate, relatively to the fragmentation. Carr and Dunwell (1999) proved under reasonable assumptions that for all $i \geq 1, c_{i}(t) \rightarrow 0$ as $t \rightarrow+\infty$.

\subsubsection{Rate of convergence}

The natural question that arises after the convergence to equilibrium is the rate of convergence. When the $H$-Theorem 2 holds, with the relative-entropy for instance, we could hope that convergence holds in this sense. The best situation would be the dissipation bounded from below by the entropy itself, i.e. along the solutions: $D(c(t)) \geq C H\left(c(t) \mid c^{\rho}\right)$ for some constant $C>0$. This leads immediately to an exponential decay of the entropy. Unfortunately this does not hold in all cases. A recent proof for $a_{i} \sim i$ is given by Cañizo et al. (2015). Another way is to bound from below the dissipation by a non-negative function $\psi$ depending on $H$, leading to

$$
\frac{d}{d t} H\left(c(t) \mid c^{\rho}\right) \leq-\psi\left(H\left(c(t) \mid c^{\rho}\right)\right) .
$$

And the problem resumes to find sub-solutions to this ordinary differential equation. This method is named entropy entropy-dissipation, because dissipation is created by entropy itself. But this method does not in general lead to exponential decay of the entropy. The first result in this direction is due to Jabin and Niethammer (2003). Let us show their result.

Theorem 4 (Rate of convergence, Jabin and Niethammer (2003)). Assume $1 \leq a_{i}=O(i), 1 \leq b_{i}=O(i)$, $\lim _{i \rightarrow+\infty} Q_{i}^{1 / i}=1 / z_{s}$ exists $\left(z_{s}>0\right)$ and that $a_{i} z_{s} \leq \min \left(b_{i}, b_{i+1}\right)$ for every $i \geq 1$. Suppose moreover that $c^{\text {in }} \in X^{+}$with mass $\rho<\rho_{s}$ (sub-critical case), with $H\left(c^{\text {in }} \mid c^{\rho}\right)<+\infty$ and there exists $\nu>0$ such that $\sum_{i \geq 1} \exp (\nu i) c_{i}^{\mathrm{in}}<+\infty$. The solution $c$ to the Becker-Döring equations $(4-5)$ on $[0,+\infty)$ with initial data $c^{\text {in }}$ satisfies, for some constant $k$ depending on $c^{\text {in }}$ and for all $t \geq 0$

$$
H\left(c(t) \mid c^{\rho}\right) \leq H\left(c^{\mathrm{in}} \mid c^{\rho}\right) \exp \left(-k t^{1 / 3}\right) .
$$


This theorem is obtain thanks to $\psi(H)=H / \ln (H)^{2}$. And the authors were able to go back from this estimate to the convergence in $\exp \left(-k t^{1 / 3}\right)$ in the strong norm of $X^{+}$. Similar results are obtained by Cañizo et al. (2015) in various cases allowing fewer hypotheses. But these results still not provide satisfactory rate of decay, with pure exponential decay. A well know theory is the stability of linear operator. If the linearized system is locally exponentially stable, we could hope that so is the full non-linear system, in a small neighborhood of the equilibrium. And we could imagine that this small neighborhood is an absorbing set, since we have Theorem 4. In fact these steps were followed by Cañizo and Lods (2013) to obtain their nice proof of the full exponential convergence stated below.

Theorem 5 (Exponential stability, Cañizo and Lods (2013)). Under the hypothesis of Theorem 4 and in addition $\lim _{i \rightarrow+\infty} a_{i+1} / a_{i}=z_{s} \cdot \lim _{i \rightarrow \infty} Q_{i+1} / Q_{i}=1$. The solution $c$ to the Becker-Döring equations $(4-5)$ on $[0,+\infty)$ with initial data $c^{\text {in }}$ satisfies, for all $t \geq 0$

$$
\left\|c(t)-c^{\rho}\right\| \leq \exp (-\lambda t)
$$

The constant $\lambda$ is completely calculable from the constant of the problem, important fact for applicability. We refer to their article (Cañizo and Lods, 2013) for a very well-detailed introduction and presentation to the result. We mention that the linear Becker-Döring system (with constant monomer $c_{1}$ ) also exhibits exponential decay Kreer (1993), and a quantitative comparison of the convergent rates will be of interest.

We finish this section pointing out that the critical case is still completely open. The super-critical too. But in this case it might not happen for the reason we will detail in the Section 2.4.

\subsection{Coarsening and relation to transport equation}

From the Becker-Döring equations (4-5), the reader familiar with numerical analysis may recognize that equations on $c_{i}$, for every $i \geq 2$, has the flavor of a discretization of a transport equation. To make the link more apparent, it is useful to write down the weak form of Eq. (4-5), which is also a very useful tool for the study of the BD system it-self. Take $\left(\varphi_{i}\right)_{i \geq 2}$ a sufficiently regular sequence, we then obtain

$$
\frac{d}{d t} \sum_{i \geq 2} c_{i}(t) \varphi_{i}=\varphi_{2} J_{1}+\sum_{i \geq 2}\left(\varphi_{i+1}-\varphi_{i}\right) J_{i}
$$

where we recall the fluxes $J_{i}$ are defined by Eq. (3). Clearly, $\left(\varphi_{i+1}-\varphi_{i}\right)$ can be seen as a discrete "spatial" derivative. Moreover, assuming some "spatial continuity", it is tempting to rewrite $J_{i}$ as $J_{i} \approx\left(a_{i} c_{1}-b_{i}\right) c_{i}$. With such ansatz, the last equation (11) then motivates the introduction of the following continuous transport equation (in a weak form)

$$
\frac{d}{d t} \int_{0}^{\infty} \varphi(x) f(t, x) d x=\varphi(0) N(t)+\int_{0}^{\infty} \varphi^{\prime}(x) j(t, x) f(t, x) d x,
$$

where the flux now reads

$$
j(t, x)=a(x) u(t)-b(x)
$$

for some appropriate functions $a$ and $b$, and a function $u$ that plays the role of $c_{1}$. We will see later what should be $N$ and what becomes the mass conservation stating $u$ in the subsequent sections. Both are the main difficulties of the problem in linking the discrete Eq. (11) to the continuous Eq. (12).

As a matter of fact, they depend crucially on the scaling hypothesis (a small parameter which allows passing from discrete size $i$ to continuous size $x$ ) and on the kinetic coefficients $a$ and $b$. We note that Eq. (12) is the weak form of a nonlinear transport equation known as the Lifshitz-Slyozov (LS) equation, after the work by Lifshitz and Slyozov (1961),

$$
\frac{\partial}{\partial t} f+\frac{\partial}{\partial x}(j(t, x) f(t, x))=0
$$

together with (if appropriate) the boundary condition at $x=0$,

$$
\lim _{x \rightarrow 0^{+}} j(t, x) f(t, x)=N(t)
$$

and an equation for $u$. Rigorous results making connection from the Becker-Döring Eq. (4-5) to the LifshitzSlyozov Eq. (13) are of two kinds. First, in the works initiated by Laurençot and Mischler (2002), Collet et al. (2002), and pursued in Deschamps et al. (2016), the authors proved that a suitable rescaling of the solution to BD equations (with the essential assumption of large excess of monomers $c_{1}$ ) converges to a solution of LS, on any finite time period and either in density or measure functional spaces. Second, in the works initiated by Penrose et al. (1978) and pursued by Penrose (1997), Niethammer (2003) and Niethammer (2004), the authors show that long-time behavior of super-critical solutions to BD equations are closed to the solution of LS. 


\subsubsection{Evolution of large clusters in the super-critical case}

We saw in Theorem 3 , in the case $\rho>\rho_{s}$, that the solution behaves particularly, as infinitly large clusters are created as time goes to infinity. The idea by Penrose (1997) is to perform a time/space scaling to approach the cluster distribution, both in a very long time and for very large sizes, in order to explain the loss of mass $\rho-\rho_{s}>0$ in the super-critical case. The formal arguments for coefficients given by Eq. (2) with $\gamma=\alpha=1 / 3$, are derived by Penrose (1997), we refer also to the review by Slemrod (2000). We present here the rigorous result obtained by Niethammer (2003), for any coefficients given by Eq. (2) where the author proved that large clusters obey a variant of LS, named the Lifshitz-Slyozov-Wagner (LSW) equations, see below. For a review on LSW and Ostwald Ripening (out of the scope of this paper), see Niethammer et al. (2006) and Niethammer (2008).

We sketch the formal arguments following Penrose (1997) and Niethammer (2003). To consider the behavior of large clusters at large time, we introduce an ad hoc small parameter $0<\varepsilon \ll 1$ such that $1 / \varepsilon$ will be a measure of a typical large cluster. Within the particular choice of coefficients given by Eq. (2), it turns that a new time scale given by $\tau=\varepsilon^{1-\alpha+\gamma} t$, where $\alpha$ and $\gamma$ are the exponents arising in the coefficients, is an appropriate time scaling to obtain a non trivial dynamics. Indeed, we obtain by the BD equations (4-5) the reformulation

$$
\frac{d}{d \tau} c_{i}=\frac{1}{\varepsilon^{1-\alpha+\gamma}}\left(J_{i-1}-J_{i}\right),
$$

and the fluxes $J_{i}$ in Eq. (3) become

$$
J_{i}=a_{i}\left(c_{1}-z_{s}-\frac{q}{i^{\gamma}}\right) c_{i}-\left(b_{i+1} c_{i+1}-b_{i} c_{i}\right),
$$

for every $i \geq 1$. Since large clusters are formed as time goes to infinity, it is possible to consider the system after a (possibly long) time $t_{\varepsilon}$ for which the relative entropy $H\left(c \mid c^{\rho_{s}}\right)$ is small enough, namely of order $\varepsilon^{\gamma}$. This suggests that the small clusters, up to some cut-off $i_{\varepsilon}$ are close to their equilibrium value, for $t \geq t_{\varepsilon}$ and $i \leq i_{\varepsilon}$, given by

$$
c_{i}(t)=Q_{i} z_{s}^{i}(1+o(1)) .
$$

On the other hand large cluster may be described by a continuous variable $x=\varepsilon i$ for $i \geq i_{\varepsilon}$. Thus, we define a density $f$ (stepwise) according to the variable $x \geq \varepsilon i_{\varepsilon}$ by

$$
f^{\varepsilon}(\tau, \varepsilon i)=\frac{1}{\varepsilon^{2}} c_{i}(\tau) .
$$

Respectively we let $u^{\varepsilon}(\tau)=\left(c_{1}(t)-z_{s}\right) / \varepsilon^{\gamma}$. This yields, after some manipulations, to

$$
\frac{\partial f^{\varepsilon}(\tau, x)}{\partial \tau}+\frac{j^{\varepsilon}(\tau, x-\varepsilon) f^{\varepsilon}(\tau, x-\varepsilon)-j^{\varepsilon}(t, x) f^{\varepsilon}(t, x)}{\varepsilon}=o(1),
$$

with $j^{\varepsilon}(\tau, x)=x^{\alpha}\left(u^{\varepsilon}(\tau)-\frac{q}{x^{\gamma}}\right)$. Formal arguments lead, as $\varepsilon \rightarrow 0$, to a solution $f$ of Eq. (13). In turns, the mass conservation (6) becomes

$$
\rho=\sum_{i=1}^{\infty} i c_{i}(\tau)=\sum_{i=1}^{i_{\varepsilon}} i c_{i}(\tau)+\sum_{i=i_{\varepsilon}}^{\infty} i c_{i}=\rho_{s}+\int_{0}^{\infty} x f(\tau, x) d x+o(1),
$$

At the limit, we obtain $\int_{0}^{\infty} x f(\tau, x) d x=\rho-\rho_{s}$ which measures large clusters formation. Such condition, complemented with the LS equation (13), allows to determined $u$ by the following expression

$$
u(\tau)=\frac{q \int_{0}^{\infty} x^{\alpha-\gamma} f(\tau, x), d x}{\int_{0}^{\infty} x^{\alpha} f(\tau, x) d x} .
$$

We now state the result obtained by Niethammer (2003).

Theorem 6 (Lifschitz-Slyozov-Wagner limit, Niethammer (2003)). Assume kinetic coefficient are given by Eq. (2), that the initial condition $c^{\varepsilon}(0)$ satisfies $H\left(c^{\varepsilon}(0) \mid c^{\rho_{s}}\right)=\varepsilon^{\gamma}$ and that $\sum_{i \geq M / \varepsilon} i c_{i}^{\varepsilon}(0) \rightarrow 0$ as $M$ goes to infinity uniformly in $\varepsilon>0$.

There is a subsequence $\left\{\varepsilon_{n}\right\}$ converging to 0 , a measure-valued function $t \mapsto \nu_{t}$ solution of $L S$ equation (13) in $\mathcal{D}^{\prime}\left(\mathbb{R}_{+} \times(0,+\infty)\right)$ such that

$$
\int_{0}^{\infty} \varphi(x) f^{\varepsilon}(\tau, x) d x \rightarrow \int_{0}^{\infty} \varphi(x) \nu_{t}(d x),
$$

locally uniformly in $t \in \mathbb{R}^{+}$, for all $\varphi \in C_{0}^{0}((0, \infty))$ and for all $t \geq 0$, and

$$
\int_{0}^{\infty} x \nu_{t}(d x)=\rho-\rho_{s}
$$


We also mention the case of vanishing small excess of density, $\rho-\rho_{s} \rightarrow 0$ as $\varepsilon \rightarrow 0$, by Niethammer (2004), where the authors recovered the LS equation, in a similar framework.

\subsubsection{Rescaled solution of BD for large monomer density}

Another point of view is to consider fast reaction rates $a_{i} c_{1} c_{i} \sim b_{i+1} c_{i+1}$ of order $1 / \varepsilon$, where $0<\varepsilon \ll 1$, together with a large excess of monomers. Namely, the characteristic number of free particles $c_{1}$ is two orders of magnitude greater than the characteristic number of clusters with size $i \geq 2$. Following Collet et al. (2002), alternatively Deschamps et al. (2016), this leads to a rescaled version of the BD equations (4-5) given, for $\varepsilon>0$, by

$$
\begin{aligned}
\frac{d}{d t} u^{\varepsilon} & =-\varepsilon J_{1}^{\varepsilon}-\varepsilon \sum_{i \geq 1} J_{i}^{\varepsilon}, \\
\frac{d}{d t} c_{i}^{\varepsilon} & =\frac{1}{\varepsilon}\left[J_{i-1}^{\varepsilon}-J_{i}^{\varepsilon}\right],
\end{aligned}
$$

for every $i \geq 1$, where $u^{\varepsilon}$ is the dimensionless version of $c_{1}$ (not to be confused with the previous section) and the scaled fluxes are

$$
J_{1}^{\varepsilon}=\alpha^{\varepsilon}\left(u^{\varepsilon}\right)^{2}-b_{2}^{\varepsilon} c_{2}^{\varepsilon}, \quad J_{i}^{\varepsilon}=a_{i}^{\varepsilon} u^{\varepsilon} c_{i}^{\varepsilon}-b_{i+1}^{\varepsilon} c_{i+1}^{\varepsilon},
$$

for every $i \geq 1$. Theorem 1 provides existence and uniqueness of solution at fixed $\varepsilon>0$. Collet et al. (2002) constructed a sequence of "density" approximations in the Lebesgue space $L^{1}\left(\mathbb{R}_{+}\right)$by, for all $t \geq 0$ and $x \geq 0$

$$
f^{\varepsilon}(t, x)=\sum_{i \geq 2} c_{i}^{\varepsilon}(t) \mathbf{1}_{\Lambda_{i}^{\varepsilon}}(x),
$$

where $\Lambda_{i}^{\varepsilon}=[(i-1 / 2) \varepsilon,(i+1 / 2) \varepsilon)$ for each $i \geq 2$. Note the first cluster is excluded from the density, it is like assuming a solute with density $f^{\varepsilon}$ belonging to the solvent $u^{\varepsilon}$ (in large excess). Then, macroscopic aggregation and fragmentation rates are constructed as functions on $\mathbb{R}_{+}$(similarly to $f^{\varepsilon}$ ), for each $\varepsilon>0$ and $x \geq 0$,

$$
a^{\varepsilon}(x)=\sum_{i \geq 2} a_{i}^{\varepsilon} \mathbf{1}_{\Lambda_{i}^{\varepsilon}}(x), \quad b^{\varepsilon}(x)=\sum_{i \geq 2} b_{i}^{\varepsilon} \mathbf{1}_{\Lambda_{i}^{\varepsilon}}(x) .
$$

This scaling supposes the first coagulation rate $\alpha^{\varepsilon}$ is faster (order $1 / \varepsilon^{2}$ ) than the other rates $a_{i}^{\varepsilon}$ for $i \geq 2$, which justifies the use of another notation $\alpha^{\varepsilon}$ and a special treatment outside the function $a^{\varepsilon}$. Theoretical justifications can be found in Collet et al. (2002). Finally, the balance of mass reads in this case, for all $t \geq 0$

$$
u^{\varepsilon}(t)+\int_{0}^{\infty} x f^{\varepsilon}(t, x) d x=\rho^{\varepsilon},
$$

for some $\rho^{\varepsilon}>0$. The value of $\rho^{\varepsilon}$ is entirely determined by the initial condition at time $t=0$.

Again we deal with the limit $\varepsilon \rightarrow 0$, and we hope the limit of $f^{\varepsilon}$ satisfies in some sense the LS equation (13). Let us introduce few hypotheses for the limit theorem, namely we assume, there exists a constant $K>0$, independent on $\varepsilon>0$, such that, for all $x \geq 0$,

$$
a^{\varepsilon}(x)+b^{\varepsilon}(x) \leq K(1+x) .
$$

Also, we assume there exists a measure $\mu^{i n}$ on $\mathbb{R}_{+}$such that

$$
\lim _{\varepsilon \rightarrow 0} \int_{0}^{\infty} \varphi(x) f^{\varepsilon}(0, x) d x=\int_{0}^{\infty} \varphi(x) \mu^{\mathrm{in}}(d x),
$$

for all $\varphi \in \mathcal{C}_{0}((0,+\infty))$ and

$$
\lim _{R \rightarrow+\infty} \sup _{\varepsilon>0} \int_{R}^{\infty} x f^{\varepsilon}(0, x) d x=0 .
$$

This estimate on the tail of the initial distribution is a classical argument which increase the compactness and will allow then to pass to the limit in the balance of mass (17).

Finally, we resume in the following the results obtained by Collet et al. (2002) in their Theorem 2.3, by Laurençot and Mischler (2002) in Theorem 2.2 for a different framework, and also modified by Deschamps et al. (2016), in Lemma 5.

Theorem 7 (Lifschitz-Slyozov limit, Collet et al. (2002), Laurençot and Mischler (2002)). Assume that $\alpha^{\varepsilon}$ is uniformly bounded, and that $a^{\varepsilon}$ and $b^{\varepsilon}$ satisfy Eq. (18). Suppose moreover that there exists $\rho \geq 0$ and two 
non-negative real functions $a$ and $b$ defined on $\mathbb{R}_{+}^{*}$ such that, when $\varepsilon$ converges to $0, \rho^{\varepsilon}$ converges to $\rho$, $a^{\varepsilon}$ and $b^{\varepsilon}$ converge locally uniformly on $\mathbb{R}_{+}^{*}$ toward, respectively, a and $b$.

If the family $\left\{f^{\varepsilon}(0, \cdot)\right\}$ satisfies Eqs. (19) and (20), then from all sequences $\left\{\varepsilon_{n}\right\}$ converging to 0 we can extract a subsequence still denoted $\left\{\varepsilon_{n}\right\}$ such that

$$
\lim _{n \rightarrow \infty} \int_{0}^{\infty} \varphi(x) f^{\varepsilon_{n}}(t, x) d x=\int_{0}^{\infty} \varphi(x) \mu(t, d x),
$$

locally uniformly in $t \in \mathbb{R}_{+}$, and for all $\varphi \in \mathcal{C}_{0}((0,+\infty))$, where $\mu:=(\mu(t, \cdot))_{t>0}$ is a measure-valued function satisfying the LS equation (13) in $\mathcal{D}^{\prime}\left(\mathbb{R}_{+} \times(0,+\infty)\right)$ where $u \in \mathcal{C}\left(\mathbb{R}_{+}\right)$is non-negative and satisfies, for all $t \geq 0$,

$$
u(t)+\int_{0}^{\infty} x \mu(t, d x)=\rho .
$$

The proof relies, mainly, on moment estimates and equicontinuity arguments. This theorem does not conclude on the full convergence of the family as $\varepsilon \rightarrow 0$. To that it requires a uniqueness argument of the limit problem Eq. (13) in measure with the balance of mass (22). Looking Eq. (13) against functions in $\mathcal{D}\left(\mathbb{R}_{+} \times(0,+\infty)\right)$ allows uniqueness with the necessary condition that the flux $j(t, x)$ points outward the domain at $x=0$ (for instance if $a(0) \rho-b(0)<0)$. We refer to the works by Niethammer and Pego (2000), Collet and Goudon (2000) and by Laurençot (2001) for the well-posedness theory on the Lifshitz-Slyozov equation. Also, we mention that the convergence in Eq. (21) has also been shown to hold in a functional density space, in $L^{1}(x d x)$, by Laurençot (2002).

We are now concerned with the case the flux $j(t, x)$ points inward the domain at $x=0$, for instance if $a(0) u(0)-b(0)>0$, or more generally if the characteristics, backward solution of

$$
\frac{d}{d t} x=j(t, x)
$$

goes back to $x=0$ in finite time. In this case, it is hopeless to obtain a well-defined limit to the LS equation (13) without a boundary condition, of type (14). A rigorous identification of the boundary condition has been performed by Deschamps et al. (2016). It was obtained through the limit of the rescaled BD equations (15-16) in the spirit of Theorem 7. More precisely, we assumed, $a(x) \sim_{0} \bar{a} x^{r_{a}}$ and $b(x) \sim_{0} \bar{b} x^{r_{b}}$ with $r_{a} \leq r_{b}$ and $r_{a}<1$. These assumptions allow a fine control of the pointwise value of the solution at $x=0$ to obtain the boundary value. The limit obtained is a measure-valued solution to LS on $[0, T]$, identifiable if $\sup _{t \in[0, T]} u(t)>$ $\lim _{x \rightarrow 0} b(x) / a(x)$ which correspond to time interval on which characteristic goes back to $x=0$. Let us present an informal version of a result we obtained.

Theorem 8 (Boundary value, Deschamps et al. (2016)). A "good" boundary condition at $x=0$ for the LifschitzSlyozov equation, when $a(x)=\bar{a} x^{r_{a}}$ and $b(x)=\bar{b} x^{r_{b}}$ with $r_{a}<1$ and $r_{a} \leq r_{b}$, is

$$
\lim _{x \rightarrow 0^{+}} j(t, x) f(t, x)= \begin{cases}\alpha u(t)^{2}, & \text { if } r_{a}<r_{b}, u(t)>0 ; \\ \frac{\alpha}{\bar{a}} u(\bar{a} u-\bar{b}), & \text { if } r_{a}=r_{b}, u(t)>\bar{b} / \bar{a},\end{cases}
$$

where $\alpha$ is the limit of $\alpha^{\varepsilon}$ as $\varepsilon$ goes to 0 . In both cases, this also reads

$$
\lim _{x \rightarrow 0^{+}} x^{r_{a}} f(t, x)=\frac{\alpha}{\bar{a}} u(t) \text {. }
$$

Note the conditions on $r_{a}, r_{b}$ and $u$ are well related to incoming characteristic. Theorems 1 and 2 by Deschamps et al. (2016) also assumed a technical growth condition (in $\varepsilon$ ) on the "relatively small" sizes, through the condition

$$
\sup _{\varepsilon>0} \sum_{i \geq 2} \varepsilon^{r_{a}} c_{i}^{\mathrm{in}, \varepsilon} e^{-i z}<+\infty
$$

for all $z \in(0,1)$. This is the key estimates which is proved to propagate in time (see Proposition 2). This allows a quasi steady-state limit of the small cluster concentrations, that behave as fast variables in Eq. (16). Note in the case of exact power law, Deschamps et al. (2016) also proved with extra reasonable assumptions on initial conditions, that the limit measure solution has a density with respect to $x^{r_{a}} d x$. Finally, other scalings of the first fragmentation rate are investigated by Deschamps et al. (2016). Also, these results do not provide a complete answer. Indeed, uniqueness for the inward case is not achieved and we are not aware if $u$ can cross the threshold $\lim _{x \rightarrow 0} b(x) / a(x)$.

Remark 1. Second-order approximations (Fokker-Planck like) of BD equations are still under intense active research, and a full satisfactory answer is still an open problem, see proposed equations by Velázquez (1998, 2000), Hariz and Collet (1999); Collet et al. (2002); Collet (2004), Conlon et al. (2016). Arbitrary higher order terms are formally derived by Niethammer (2003). 


\subsection{Time-dependent properties, Metastability and Classical nucleation theory}

The following properties are of the most important ones in application of the BD equations to phase transition. Yet, as for the convergence rate to equilibrium, coarsening and evolution of large sized clusters, available results are still incomplete. The main result we are aware of on metastability for BD equations (4-5) is given by Penrose (1989). The ideas of classical nucleation theory goes back to Becker and Döring (1935), and is built on the remark that there exist steady-state solutions of the Eqs. (5) (with $c_{1}$ constant) with non-zero steady-state flux, which can be arbitrary small in some sense. This very small steady-state flux is interpreted as the rate of formation of larger and larger cluster, leading to a phase transition phenomena in long time. The term metastability in such theory refers to the fact that the rate is arbitrary small. Penrose (1989) goes much beyond by extending this notion of metastability to a time-dependent phenomenon (instead of a steady-state one). Indeed, he could exhibit a solution of the full system (4-5) that enters a state that lived for exponentially long time, yet can be distinguished from the equilibrium state. This solution is a super-critical solution, with $\rho>\rho_{s}$, and is required to have a well prepared initial condition. This solution is also related in some sense to an extremely small common flux value. It remains an important open question to know whether the metastable state can be reached from a larger class of initial data.

Penrose (1989) considered technical conditions on coefficients which are essentially satisfied by the ones given by Eq. (2). The crucial initial condition is then constructed as follows. For any $z>z_{s}$, let $f_{i}(z)$ be the unique solution of

$$
a_{i-1} z f_{i-1}(z)-\left(b_{i}+a_{i} z\right) f_{i}(z)+b_{i+1} f_{i+1}(z)=0, \quad i \geq 2,
$$

with end conditions $f_{1}(z)=z$ and $\sup _{i} f_{i}(z)<\infty$. Actually, $f_{i}$ can be solved explicitly by (for $z>z_{s}$ the reader can check that the infinite series are convergent)

$$
f_{i}(z)=J(z) Q_{i} z^{i} \sum_{r=i}^{\infty} \frac{1}{a_{i} Q_{i} z^{i+1}}, \quad J(z):=\left[\sum_{r=1}^{\infty} \frac{1}{a_{i} Q_{i} z^{i+1}}\right]^{-1} .
$$

Let $i^{*}$ be the critical cluster size defined as the (unique) size that minimizes the quantity $a_{i} Q_{i} z^{i}$. The metastable state exhibited by Penrose (1989) has to be understood in the limit of small excess of density, $z \searrow z_{s}$. The following terminology is used

- $g(z)$ is exponentially small if for each $m>0, g(z)=O\left(\left(z-z_{s}\right)^{m}\right)$.

- $g(z)$ is at most algebraically large if for some $m>0, g(z)=O\left(\left(z-z_{s}\right)^{-m}\right)$.

The main theorem by Penrose (1989) reads

Theorem 9 (Metastability, Penrose (1989)). Let c be the solution of the BD Eqs. (4)-(5) with initial condition

$$
c_{i}(0)= \begin{cases}f_{i}(z), & \text { if } \quad i \leq i^{*} \\ Q_{i} z_{s}^{i}, & \text { if } \quad i>i^{*}\end{cases}
$$

Then $c$ has an exponentially long lifetime as $z \searrow z_{s}$, in the sense that for each fixed $i$ (note that $\left.i^{*} \rightarrow \infty\right)$ :

- if $t$ is at most algebraically large, then $c_{i}(t)-c_{i}(0)$ is exponentially small

- $\lim _{t \rightarrow \infty}\left[c_{i}(t)-c_{i}(0)\right]$ is not exponentially small

Thus, cluster with size $i \ll i^{*}$ remain exponentially close to their initial values, until an exponentially long time has elapsed. But eventually they do change. Note that the initial values for the small clusters, $f_{i}(z)$, correspond to the steady-state values of the classical nucleation theory, for which $J_{i-1}(0)=J_{i}(0)$ for all $2 \leq i<i^{*}$, and the common flux value is $J(z)$, which is also exponentially small as $z \searrow z_{s}$. We refer the reader to (Penrose, 1989, Theorems 1 and 2) for orders of magnitude of $i^{*}, J(z)$ and quantification of the (small) growth rate of large clusters of size greater than $i^{*}$.

Remark 2. The numerical illustration of the metastability is a problem per se, we refer the reader to the two nice papers by Carr et al. (1995) and by Duncan and Soheili (2001), where numerical schemes are derived and are shown to consistently represent the metastable states. The reader may also look at the section 3.4 where numerical simulations of the stochastic Becker-Döring are shown. Finally, let us mention that analogous metastability properties have been investigated in the classical linear version of BD by Penrose (1989) and Kreer (1993), in a finite-dimensional truncated version by Dunwell (1997) and Duncan and Dunwell (2002), and in a thermodynamically consistent version of the BD system by Ssemaganda and Warnecke (2013). 


\section{$3 \quad$ Stochastic Becker-Döring model}

Due to space considerations, we will not detail historical facts on the study of stochastic coagulation-fragmentation models. Let us just mention that the first study of a stochastic coagulation models is widely attributed to Marcus (1968) and Lushnikov (1978) which give the name to the Marcus-Lushnikov process, stochastic analog of the pure coagulation Smoluchowski's equations. Up to our knowledge, Whittle (1965) and Kelly (1979) are pioneering in the study of more general stochastic coagulation-fragmentation models (including the Becker-Döring model). See Aldous (1999) and the discussion in Freiman and Granovsky (2005) for more details.

\subsection{Definition and State-space}

A stochastic version of the Becker-Döring model may be defined as a continuous time Markov chain analog of the set of ordinary differential equations (4-5), for which transition are given by the same set of kinetic reactions (1), but modeling discrete numbers of clusters instead of continuous concentrations. Precisely, given a positive integer $M$, we define the state space

$$
X_{M}:=\left\{C=\left(C_{i}\right)_{i \geq 1} \in \mathbb{N}^{\mathbb{N}}: \sum_{i=1}^{M} i C_{i}=M\right\} .
$$

On $X_{M}$, we introduced the following operators defined by, for any configuration $C$ on $X_{M}$,

$$
\begin{aligned}
& R_{1}^{+} C=\left(C_{1}-2, C_{2}+1, \cdots, C_{i}, \cdots\right) \\
& R_{2}^{-} C=\left(C_{1}+2, C_{2}-1, \cdots, C_{i}, \cdots\right)
\end{aligned}
$$

and, for any $i \geq 2$,

$$
\begin{aligned}
R_{i}^{+} C & =\left(C_{1}-1, C_{2}, \cdots, C_{i}-1, C_{i+1}+1, \cdots\right) \\
R_{i+1}^{-} C & =\left(C_{1}+1, C_{2}, \cdots, C_{i}+1, C_{i+1}-1, \cdots\right)
\end{aligned}
$$

Given non-negative kinetic rates $\left(a_{i}\right)_{i \geq 1},\left(b_{i}\right)_{i \geq 2}$, the stochastic Becker-Döring model (SBD) is defined as the continous time Markov chain on $X_{M}$ with transition rates

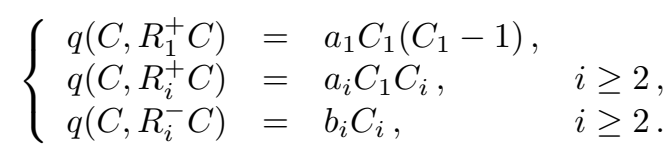

Given an initial configuration $C^{\text {in }} \in X_{M}$ (deterministic or random), the configuration $C(t)$ defined by the SBD may alternatively be represented as the solution of the following system of stochastic equations

$$
\left\{\begin{array}{l}
C_{1}(t)=C_{1}^{\text {in }}-2 J_{1}(t)-\sum_{i \geq 2} J_{i}(t) \\
C_{i}(t)=C_{i}^{\text {in }}+J_{i-1}(t)-J_{i}(t), \quad i \geq 2
\end{array}\right.
$$

with

$$
J_{i}(t)=Y_{i}^{+}\left(\int_{0}^{t} a_{i} C_{1}(s)\left(C_{i}(s)-\delta_{1, i}\right) d s\right)-Y_{i+1}^{-}\left(\int_{0}^{t} b_{i+1} C_{i+1}(s) d s\right), \quad i \geq 1,
$$

where $\delta_{1, i}=1$ if $i=1$ and $\delta_{1, i}=0$ if $i>1$ and $Y_{i}^{+}, Y_{i+1}^{-}$for $i \geq 1$ are independent standard Poisson processes. Analogy between Eq. (23) and Eq. (4-5) is clear. The number of clusters of size $i \geq 2$ evolves according to the differences between two (stochastic) cumulative counts $J_{i-1}$ and $J_{i}$. Finally, we may also identified the SBD with the help of its infinitesimal generator $L_{M}$, defined by, for any bounded functions $f$ on $X_{M}$,

$$
L_{M} f(C)=\sum_{i=1}^{M-1}\left[f\left(R_{i}^{+} C\right)-f(C)\right] a_{i} C_{1}\left(C_{i}-\delta_{1, i}\right)+\left[f\left(R_{i+1}^{-} C\right)-f(C)\right] b_{i+1} C_{i+1} .
$$

Thanks to the Markov processes theory, we deduce in particular that, for any bounded functions $f$ on $X_{M}$,

$$
f(C(t))-f\left(C^{\mathrm{in}}\right)-\int_{0}^{t} L_{M} f(C(s)) d s
$$

is a centered martingale, and, taking $f_{C}\left(C^{\prime}\right)=\mathbf{1}_{\left\{C^{\prime}=C\right\}}$, we deduce the following Backward kolmogorov equation on the probability $P(t, \cdot)$ on $X_{M}$ (Master equation)

$$
\begin{aligned}
\frac{d}{d t} P(t ; C)=\sum_{i=1}^{M-1} a_{i}\left(C_{1}+1\right)\left(C_{i}+1+\delta_{1, i}\right) P\left(t ; R_{i+1}^{-} C\right)- & a_{i} C_{1}\left(C_{i}-\delta_{1, i}\right) P(t ; C) \\
& +\sum_{i=2}^{M} b_{i}\left(C_{i}+1\right) P\left(t ; R_{i-1}^{+} C\right)-b_{i} C_{i} P(t ; C)
\end{aligned}
$$


Although the well-posedness of the SBD model is of course standard (as a pure-jump Markov process on a finite state-space), a first non trivial question arises with respect to the precise description of the state space, and in particular to its cardinality. In fact, the state space $X_{M}$ is given by all possible partitions of the integer $M$, a well-known problem in combinatorics. In particular, one can show the recurrence formula and the asymptotic as $M \rightarrow \infty$, (Flajolet and Sedgewick, 2009, chap I.3) ${ }^{1}$

$$
M\left|X_{M}\right|=\sum_{i=1}^{M} \sigma(i)\left|X_{M-i}\right|, \quad\left|X_{M}\right| \propto \frac{1}{4 M \sqrt{3}} \exp \left(\pi \sqrt{\frac{2 M}{3}}\right),
$$

where $\sigma(i)$ is the sum of the divisors of $i$ (e.g. $\sigma(6)=1+2+3+6=12$ ).

Remark 3. We mention that some terminology in the literature may be confusing. Indeed, some authors Bhakta and Ruckenstein (1995) have named the deterministic Becker-Döring system (4-5) a stochastic version of the Lifshitz-Slyozov(-Wagner) equations. Such terminology seems to be motivated by the fact that the size of clusters are modeled as discrete variable in Eq. (4-5), and that such system has the "flavor" of a master equation for a random walk in $\mathbb{N}^{+}$.

Remark 4. As for the BD system (4-5), some variants have been considered for the SBD. Let us mention for instance the constant monomer system studied in Yvinec et al. (2016) (which leads to a Poissonian equilibrium distribution), the exchange-driven growth model in Ben-Naim and Krapivsky (2003) (where clusters exchange monomer in one single step), some reduced version for specific kinetic rates $(a(i)=i, b(i)=0)$ see Doumic et al. (2016) and Eugene et al. (2016) or for fixed number of clusters (only one or two clusters can be present) Penrose (2008), Rotstein (2015) and Yvinec et al. (2016). Of course, the SBD can be seen as a particular case of more general coagulation-fragmentation processes Bertoin (2006). However, due to its specificity, it seems that some of the results available on general coagulation-fragmentation processes are not straightforwardly applicable (or do not bring interesting conclusions). Finally, although out of the scope of this survey, let us mention the interesting links between the (stochastic) BD system with some lattice models Chau et al. (2015), Dehghanpour and Schonmann (1997), den Hollander et al. (2000), Bovier et al. (2010), Ercolani et al. (2014), in particular for nucleation and phase transition.

\subsection{Long-time behavior}

Although the equation (24) is linear with respect to $P(t, \cdot)$, the size of the state space being exponentially large as $M \rightarrow \infty$, it is illusory to obtain a full exact solution of Eq. (24). Yet, perhaps surprisingly, the stationary solution of Eq. (24) has a relatively simple form, namely a product-form Anderson et al. (2010). Indeed, the (unique) stationary probability $\Pi$ on $X_{M}$ of Eq. (24) is given by (Kelly, 1979, Theorem 8.1)

$$
\Pi(C)=B_{M} \prod_{i=1}^{M} \frac{\left(Q_{i}\right)^{C_{i}}}{C_{i} !}
$$

where $B_{M}$ is a normalizing constant and $Q_{i}$ is defined by Eq. (8). One may verify simply that the following detailed balance condition holds (Kelly, 1979, Theorem 1.2)

$$
\Pi(C) q\left(C, R_{i}^{+} C\right)=\Pi\left(R_{i}^{+} C\right) q\left(R_{i}^{+} C, C\right)
$$

Note also that, for all $z>0$, with $B_{z}:=B_{M} / z^{M}$, the expression (25) may be rewritten $\Pi(C)=B_{z} \prod_{i=1}^{M} \frac{\left(Q_{i} z^{i}\right)^{C_{i}}}{C_{i} !}$, which has a clearer analogy with the deterministic equilibrium of the BD equation. Finally, the distribution $\Pi$ has the following probabilistic meaning: let $Z_{i}, i=1, \cdots, M$, be independent Poisson random variables with respective means $Q_{i}$, then it is easily seen that, for all $C \in X_{M}$,

$$
\Pi(C)=\mathbf{P}\left\{Z_{1}=C_{1}, \cdots, Z_{M}=C_{M} \mid \sum_{i=1}^{M} i Z_{i}=M\right\} .
$$

For the stationary distribution $\Pi$, the expected number of clusters of size $i$ is

$$
\mathbf{E}_{\Pi} C_{i}=Q_{i} B_{M} / B_{M-i}
$$

and the probability that a randomly chosen particle lies in a cluster of size $i$ is $i Q_{i} B_{M} / M B_{M-i}$, from which we deduce that the normalizing constant $B_{M}$ satisfies the recursive formula (with $B_{0}=1$ )

$$
M B_{M}^{-1}=\sum_{i=1}^{M} i Q_{i} B_{M-i}^{-1}
$$

\footnotetext{
${ }^{1}$ R.Y thanks Bence Melykuti for pointing out this fact
} 
Moreover, $B_{M}^{-1}$ is the coefficient of $z^{M}$ in the power series expansion of

$$
G(z)=\exp \left(\sum_{i} Q_{i} z^{i}\right)
$$

Remark 5. In some examples, the recursive formula (26) may be solved exactly. For instance, if $a_{i}=a i, b_{i}=b i$, then the equilibrium probability is given by the closed-form formula

$$
\Pi(C)=\left(\begin{array}{c}
b / a+M-1 \\
M
\end{array}\right)^{-1} \prod_{i=1}^{M} \frac{1}{C_{i} !}\left(\frac{b}{a i}\right)^{C_{i}}
$$

Besides the analytical form of the equilibrium distribution $\Pi$, it is a natural question to ask what is its limiting behavior as $M \rightarrow \infty$. Under the assumption that

$$
\lim _{i \rightarrow \infty} \frac{a_{i}}{b_{i+1}}=z_{s}>0
$$

(which is slightly stronger than the hypothesis on $Q_{i}^{1 / i}$ used in Theorem 2 and 3), one can show Freiman and Granovsky (2002); Bell and Burris (2003) that $G$ has also for radius of convergence $z_{s}$, and in such case, the expected number of clusters of size $i$ has a limit as $M \rightarrow \infty$, given by

$$
\lim _{M \rightarrow \infty} \mathbf{E}_{\Pi} C_{i}=Q_{i} z_{s}^{i}
$$

Other functionals of the stationary distribution $\Pi$ have been derived by Durrett et al. (1999). In particular, let us mention that the variance of $C_{i}$, under $\Pi$ and with hypothesis (27), satisfies the same asymptotic relation (28), and that $C_{i}, C_{j}, i \neq j$, becomes asymptotically uncorrelated as $M \rightarrow \infty$. It is also interesting to note the link of the limit (28) with the supersaturation case in the deterministic BD theory, see Theorem 3. Study of the limit shape of the stationary distribution $\Pi$ (and quantities like the size of the largest or lowest component) is a well-known problem in statistical physics or in combinatorics (study of random integer partitions and Young diagrams) and goes back to Khinchin's probabilistic method Khinchin (1960). Detailed description of such field is out of the scope of this survey, and we refer the reader to Freiman and Granovsky (2005), Erlihson and Granovsky (2008), Han et al. (2008), Granovsky (2013), Ercolani et al. (2014) for recent results.

In contrast to the deterministic theory, we are not aware of any work quantifying the speed of convergence toward the equilibrium distribution (25) (which has to be exponential). In particular, it would be interesting to study how this rate behave as $M \rightarrow \infty$.

Remark 6. Strong binding limit for constant coefficients has been considered in D'Orsogna et al. (2012) (linked to the almost pure-coagulation deterministic dynamics in King and Wattis (2002)) and illustrates how mass incommensurability arises for finite mass $M$, when a fixed maximal cluster size $N<M$ is further imposed.

\subsection{Large Number and relation to deterministic Becker-Döring}

A first natural question when comparing the SBD and the BD system, is that can we recover the deterministic equations in the limit $M \rightarrow+\infty$ ? The main tool to answer such question is the tightness of stochastic processes, which provides an appropriate compactness property for a sequence of rescaled solutions of the SBD. As a particular case, Jeon (1998) has considered the sequence of stochastic processes $\left\{C^{n}(t)\right\}$ in $X_{n}^{+}:=\left\{\frac{1}{n} C: C \in \mathbb{N}^{\mathbb{N}}, \sum_{i \geq 1} i C_{i}=n\right\} \subset X^{+} \subset l^{2}$, defined by the generator

$$
L^{n} f(C)=n \sum_{i=1}^{n}\left[f\left(R_{i, n}^{+} C\right)-f(C)\right] a_{i} C_{1}\left(C_{i}-\delta_{1, i}\right)+\left[f\left(R_{i+1, n}^{-} C\right)-f(C)\right] b_{i+1} C_{i+1},
$$

where, for all $i \geq 1$,

$$
\begin{aligned}
R_{i, n}^{+} C & =\left(C_{1}-1 / n, C_{2}, \cdots, C_{i}-1 / n, C_{i+1}+1 / n, \cdots\right) \\
R_{i+1, n}^{-} C & =\left(C_{1}+1 / n, C_{2}, \cdots, C_{i}+1 / n, C_{i+1}-1 / n, \cdots\right)
\end{aligned}
$$

Under such classical scaling (which satisfies the system size expansion), one can prove

Theorem 10 (Law of Large Numbers, Jeon (1998)). If $a(i), b(i)$ are such that

$$
\sup _{C \in X^{+}: \sum i C_{i} \leq 1} \sum_{i \geq 1} a(i) C_{i}<\infty, \quad \sup _{C \in X^{+}: \sum i C_{i} \leq 1} \sum_{i \geq 1} b(i) C_{i}<\infty,
$$

then the laws of the stochastic process $\left\{C^{n}(t)\right\}$ defined by Eq.(29) form a tight sequence as a càdlàg process in $l^{2}$. 
Note that hypothesis (30) is trivially satisfied for sublinear function of $i$. Also, it is clear that any weak limit of $\left\{C^{n}(t)\right\}$ is a solution of the BD system (4-5), which is an alternative proof of existence of solution of the BD system. Finally, convergence of the whole sequence may be obtained with the uniqueness result stated in Theorem 1.

We are not aware of any rigorous derivation of a second-order approximation of such limit, which should reasonably be a langevin stochastic differential equation version of the BD system (4-5).

\subsection{Time-dependent properties, Metastability and stochastic nucleation theory}

Up to our knowledge, the early work Schweitzler et al. (1988) paves the way to study fluctuations of the timedependent cluster distributions and first passage time in stochastic finite system nucleation models. Using physical arguments, they investigated reaction rates of the form $a_{i} \approx i^{2 / 3}$ and $b_{i} \approx i^{2 / 3} y_{0} e^{q i^{-1 / 3}}$, which are asymptotically similar to Eq. (2) (which $\alpha=2 / 3, \gamma=1 / 3$ ). One can notice that for such coefficients, a (time-dependent) critical cluster size $i_{c}(t)$ exists, defined by

$$
a_{i} C_{1}(t)-b_{i}<0, \forall i<i_{c}, \quad a_{i} C_{1}(t)-b_{i} \geq 0, \forall i \geq i_{c} .
$$

This observation has led Schweitzler et al. (1988) to analyze the SBD with the Ostwald ripening theory in mind. Specifically, with the help of numerical simulations, and heuristically derived moment closure approximation of the master equation (24) governing the clusters' distribution evolution (which resemble second-order approximation of the deterministic BD system, see Remark 1), the authors put in evidence the existence of a (stochastic) metastable state which is reached before the equilibrium distribution. Indeed, starting from an initial pure-monomer condition, on can observe a rapid transient that lead to a relatively small cluster distribution (with support contained among the size below the critical size), which has a long-lived state. Only after a first critical cluster is formed, the cluster size distribution is bimodal, given by a mixture of undercritical and overcritical clusters, until a single large cluster emerges from a competition between overcritical clusters, and its further growth is at the expense of the other clusters which now shrink. We have reproduced similar numerical simulations, with kinetic coefficients given by Eq. (2), in Fig 1 and 2.

A key event in exiting the metastable state is thus the formation of an overcritical cluster. Such event may be analyzed with the help of the first passage time theory. It is important to note that, in agreement with classical metastability theory, the authors of this previous work noticed that the first time needed to form an overcritical cluster was subjected to large fluctuations. We are not aware of any theoretical work on the metastability for the SBD system, but we may mention that several groups have recently investigated numerically the behavior of first passage time (or related quantities) in the SBD system (or related models) Bhatt and Ford (2003), Yvinec et al. (2012), Yvinec et al. (2016), Penrose (2008), Johansson (2016). In particular, it is tempting to use first passage time theory to define a stochastic analog of the so-called nucleation rate in the classical nucleation theory (see section 2.4). However, we notice that the analytical form of such nucleation rate is unclear. In particular, what should be the quasi-stationary distribution, stochastic analogous to the metastable state derived in Section 2.4 ?

Finally, let us mention the link with the study of the stochastic gelation time in Smoluchowsky's coagulation model, which has recently been the subject of active research. Let us defined, for $\alpha \leq 1$,

$$
\tau_{n}^{\alpha}=\inf \left\{t>0: C_{k}^{n}(t)>0, \text { for some } k>\alpha n\right\},
$$

where $\left\{C^{n}(t)\right\}$ is the rescaled stochastic process defined its generator in Eq. (29). It is known that for the stochastic smoluchowsky's coagulation model (see Jeon (1998), Eibeck and Wagner (2001), Fournier and Giet (2004), Fournier and Laurençot (2009), Rezakhanlou (2013), Wagner (2005)), and for specific coagulation kernel, the sequence of first passage time (31) has a finite (zero or positive) limit as $n \rightarrow \infty$, and that the limit is linked to the gelation (loss of mass) in the deterministic Smoluchowsky's coagulation model. According to the longtime behavior theory for the deterministic Becker-Döring model, it is to be expected that for the SBD, such first passage time (31) can only have infinite limit. However, rate of divergence and summary statistics (mean, variance) as $n \rightarrow \infty$ are important open questions. 

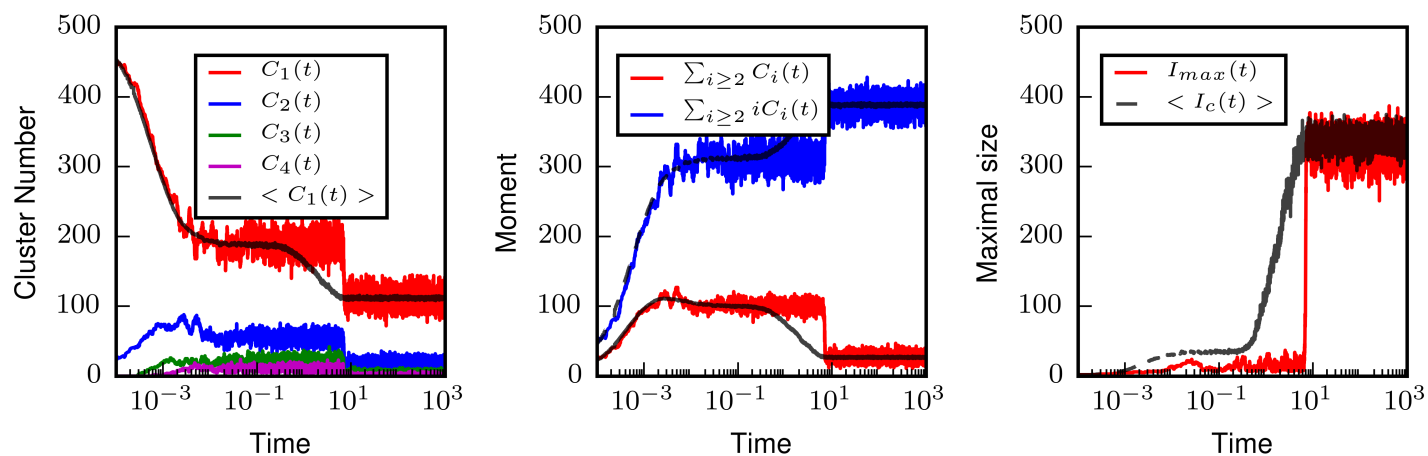

Figure 1: Time trajectories of the SBD system (23) with kinetic coefficient given by (2), with $M=500$, $\alpha=2 / 3, \gamma=1 / 3, z_{s}=500 / 11, q=10 / 11$. On the left, we plot a stochastic realization of the number of Monomers, Dimers, Tri-mers and 4-mers, together with the sampled average over 100 realizations for the number of Monomers. On the middle, we plot the total mass in clusters and their numbers, and on the right, the maximal cluster size
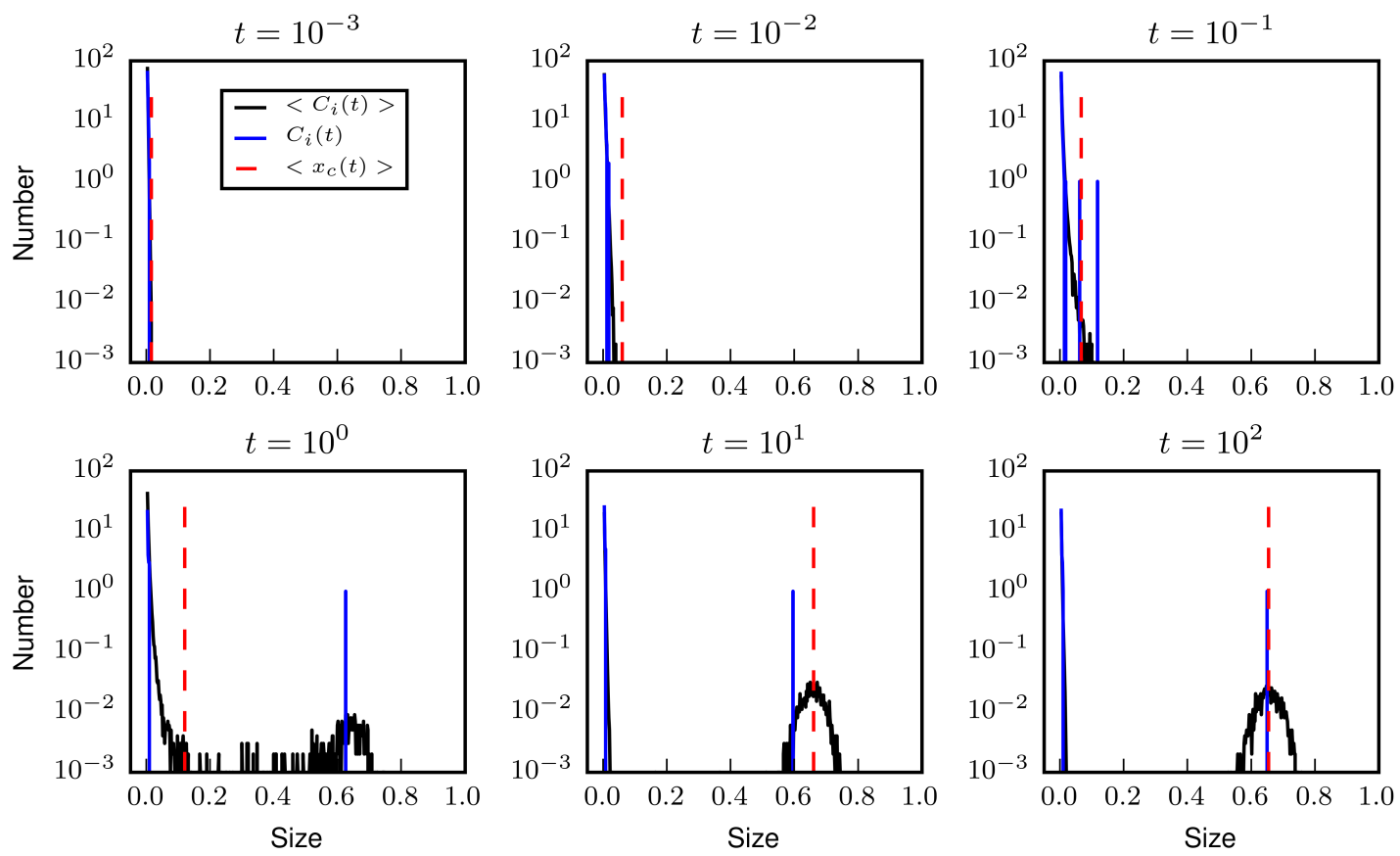

Figure 2: Cluster size distribution at distinct times $t$, corresponding to Fig 1 . The sizes are rescaled by $M$, that is $x=2 / M, \cdots, 1$. In blue we plot the distribution of a stochastic realization, in black we represent the sampled averaged distribution over 1000 realizations, and in red we plot the (rescaled) critical size $x_{c}=I_{c} / M$. 


\section{Acknowledgment}

E.H. thanks the financial support of CAPES/IMPA Brazil during the post-doc at the Universidade Federal de Campina Grande (Paraiba). R.Y thanks the Isaac Newton Institute for Mathematical Sciences, Cambridge, for support and hospitality during the programme Stochastic Dynamical Systems in Biology: Numerical Methods and Applications, where part of this work was undertaken.

\section{References}

D. J. Aldous. Deterministic and Stochastic Models for Coalescence (Aggregation and Coagulation): A Review of the Mean-Field Theory for Probabilists. Bernoulli, 5(1):3-48, 1999.

M.-T. Alvarez-Martinez, P. Fontes, V. Zomosa-Signoret, J.-D. Arnaud, E. Hingant, L. Pujo-Menjouet, and J.-P. Liautard. Dynamics of polymerization shed light on the mechanisms that lead to multiple amyloid structures of the prion protein. BBA-Proteins Proteom., 1814(10):1305-1317, October 2011.

D. F. Anderson, G. Craciun, and T. G. Kurtz. Product-form stationary distributions for deficiency zero chemical reaction networks. Bull. Math. Biol., 72(8):1947-1970, 2010.

J. M. Ball and J. Carr. Asymptotic behaviour of solutions to the Becker-Döring equations for arbitrary initial data. Proc. Roy. Soc. Edinburgh Sect. A, 108(1-2):109-116, 1988.

J. M. Ball, J. Carr, and O. Penrose. The Becker-Döring cluster equations: basic properties and asymptotic behaviour of solutions. Comm. Math. Phys., 104(4):657-692, 1986.

R. Becker and W. Döring. Kinetische Behandlung der Keimbildung in Übersättigten Dämpfen. Ann. Phys., 416(8):719-752, 1935.

J. P. Bell and S. N. Burris. Asymptotics for logical limit laws: when the growth of the components is in an RT class. Trans. Amer. Math. Soc., 355(9):3777-3794, 2003.

E. Ben-Naim and P. L. Krapivsky. Exchange-driven growth. Phys. Rev. E, 68(3), 2003.

J. Bertoin. Random fragmentation and coagulation processes, volume 102 of Cambridge Studies in Advanced Mathematics. Cambridge University Press, Cambridge, 2006.

A. Bhakta and E. Ruckenstein. Ostwald ripening: A stochastic approach. J. Chem. Phys., 103(16):7120, 1995.

J. S. Bhatt and I. J. Ford. Kinetics of heterogeneous nucleation for low mean cluster populations. J. Chem. Phys., 118(3166): 3166-3166, 2003.

A. Bovier, F. den Hollander, and C. Spitoni. Homogeneous nucleation for Glauber and Kawasaki dynamics in large volumes at low temperatures. Ann. Probab., 38(2):661-713, 2010.

P. C. Bressloff. Aggregation-fragmentation model of vesicular transport in neurons. J. Phys. A, 49(14):145601-16, 2016.

Z. Budrikis, G. Costantini, C. A. La Porta, and S. Zapperi. Protein accumulation in the endoplasmic reticulum as a non-equilibrium phase transition. Nature Communications, 5, 2014.

J. J. Burton. Nucleation Theory. In B. J. Berne, editor, Statistical Mechanics: Part A: Equilibrium Techniques, volume 5 of Modern Theoretical Chemistry, pages 195-234. Springer US, Boston, MA, 1977.

J. A. Cañizo. Convergence to equilibrium for the discrete coagulation-fragmentation equations with detailed balance. J. Stat. Phys., 129(1):1-26, 2007.

J. A. Cañizo and B. Lods. Exponential convergence to equilibrium for subcritical solutions of the Becker-Döring equations. J. Differential Equations, 255(5):905-950, 2013.

J. A. Cañizo, A. Einav, and B. Lods. Trend to Equilibrium for the Becker-Döring Equations: An Analogue of Cercignani's Conjecture. arXiv, (1509.07631):1-41, 2015.

J. Carr and F. P. da Costa. Asymptotic behavior of solutions to the coagulation-fragmentation equations. II. Weak fragmentation. J. Statist. Phys., 77(1-2):89-123, 1994.

J. Carr and R. M. Dunwell. Asymptotic behaviour of solutions to the Becker-Döring equations. Proc. Edinburgh Math. Soc. (2), 42(2):415-424, 1999.

J. Carr, D. B. Duncan, and C. H. Walshaw. Numerical approximation of a metastable system. IMA J. Numer. Anal., 15(4): 505-521, 1995.

C. Cercignani. Mathematical methods in kinetic theory. Plenum Press, New York, second edition, 1990.

Y.-X. Chau, C. Connaughton, and S. Grosskinsky. Explosive condensation in symmetric mass transport models. J. Stat. Mech.Theory E., 2015(11):P11031, 2015.

J. F. Collet. Some modelling issues in the theory of fragmentation-coagulation systems. Commun. Math. Sci., 2(suppl. 1):35-54, 2004.

J. F. Collet and T. Goudon. On solutions of the Lifshitz-Slyozov model. Nonlinearity, 13(4):1239-1262, 2000. 
J. F. Collet, T. Goudon, F. Poupaud, and A. Vasseur. The Beker-Döring system and its Lifshitz-Slyozov limit. SIAM J. Appl. Math., 62(5):1488-1500, 2002.

J. G. Conlon, M. Dabkowski, and J. Wu. On large time behavior and selection principle for a diffusive Carr-Penrose model. J. Nonlinear Sci., 26(2):453-518, 2016.

P. V. Coveney and J. A. D. Wattis. Analysis of a generalized becker-doring model of self-reproducing micelles. Proc. R. Soc. Lond. Ser. A Math. Phys. Eng. Sci., 452(1952):2079-2102, 1996.

J. K. Davis and S. S. Sindi. Initial condition of stochastic self-assembly. Phys. Rev. E, 93(2), 2016.

P. Dehghanpour and R. H. Schonmann. Metropolis dynamics relaxation via nucleation and growth. Comm. Math. Phys., 188(1): 89-119, 1997.

F. den Hollander, E. Olivieri, and E. Scoppola. Metastability and nucleation for conservative dynamics. J. Math. Phys., 41(3): $1424-1498,2000$.

J. Deschamps, E. Hingant, and E. Yvinec. Quasi steady state approximation of the small clusters in Becker-Döring equations leads to boundary conditions in the Lifshitz-Slyozov limit. arXiv, (1605.08984):1-29, 2016.

M. Doumic, S. Eugene, and P. Robert. Asymptotics of stochastic protein assembly models. arXiv, (1603.06335):1-19, 2016

D. B. Duncan and R. M. Dunwell. Metastability in the classical truncated Becker-Döring equations. Proc. Edinb. Math. Soc. (2), 45(3):701-716, 2002.

D. B. Duncan and A. R. Soheili. Approximating the Becker-Döring cluster equations. Appl. Numer. Math., 37(1-2):1-29, 2001.

R. M. Dunwell. The Becker-Doring cluster equations. PhD thesis, Heriot-Watt University, Edinburgh, 1997.

R. Durrett, B. L. Granovsky, and S. Gueron. The equilibrium behavior of reversible coagulation-fragmentation processes. J. Theoret. Probab., 12(2):447-474, 1999.

M. R. D’Orsogna, G. Lakatos, and T. Chou. Stochastic self-assembly of incommensurate clusters. J. Chem. Phys., 136(8):084110, 2012.

K. Eden, R. Morris, J. Gillam, C. E. MacPhee, and R. J. Allen. Competition between primary nucleation and autocatalysis in amyloid fibril self-assembly. Biophys. J., 108(3):632-643, 2015.

A. Eibeck and W. Wagner. Stochastic particle approximations for smoluchowski's coagulation equation. Ann. Appl. Proba., 11(4): $1137-1165,2001$.

N. M. Ercolani, S. Jansen, and D. Ueltschi. Random partitions in statistical mechanics. Electron. J. Probab., $19,2014$.

M. M. Erlihson and B. L. Granovsky. Limit shapes of gibbs distributions on the set of integer partitions: The expansive case. Ann. Inst. Henri Poincaré Probab. Stat., 44(5):915-945, 2008.

M. Escobedo, P. Laurençot, S. Mischler, and B. Perthame. Gelation and mass conservation in coagulation-fragmentation models. J. Differential Equations, 195(1):143-174, 2003.

S. Eugene, W.-F. Xue, P. Robert, and M. Doumic-Jauffret. Insights into the variability of nucleated amyloid polymerization by a minimalistic model of stochastic protein assembly. J. Chem. Phys., 144(17):175101, 2016.

P. Flajolet and R. Sedgewick. Analytic Combinatorics. Cambridge University Press, Cambridge, 2009.

N. Fournier and J.-S. Giet. Convergence of the Marcus-Lushnikov process. Methodol. Comput. Appl. Probab., 6(2):219-231, 2004.

Nicolas Fournier and Philippe Laurençot. Marcus-Lushnikov processes, Smoluchowski's and Flory's models. Stochastic Process. Appl., 119(1):167-189, 2009.

G. A. Freiman and B. L. Granovsky. Asymptotic formula for a partition function of reversible coagulation-fragmentation processes. Israel J. Math., 130(1):259-279, 2002.

G. A. Freiman and B. L. Granovsky. Clustering in coagulation-fragmentation processes, random combinatorial structures and additive number systems: Asymptotic formulae and limiting laws. Trans. Amer. Math. Soc., 357(6):2483-2507, 2005.

B. L. Granovsky. Asymptotics of counts of small components in random structures and models of coagulation-fragmentation. ESAIM Probab. Stat., 17:531-549, 2013.

D. Han, X. S. Zhang, and W. A. Zheng. Subcritical, critical and supercritical size distributions in random coagulation-fragmentation processes. Acta Math. Sin. (Engl. Ser.), 24(1):121-138, 2008.

S. Hariz and J. F. Collet. A modified version of the Lifshitz-Slyozov model. Appl. Math. Lett., 12(1):81-85, 1999.

N. Hoze and D. Holcman. Coagulation-fragmentation for a finite number of particles and application to telomere clustering in the yeast nucleus. Phys. Lett. A, 376(6):845-849, 2012.

N. Hoze and D. Holcman. Modeling capsid kinetics assembly from the steady state distribution of multi-sizes aggregates. Phys. Lett. A, 378(5):531-534, 2014.

N. Hoze and D. Holcman. Kinetics of aggregation with a finite number of particles and application to viral capsid assembly. $J$. Math. Biol., 70:1685-1705, 2015. 
J. Hu and H. G. Othmer. A theoretical analysis of filament length fluctuations in actin and other polymers. J. Math. Biol., 63: 1001-1049, 2011.

P. E. Jabin and B. Niethammer. On the rate of convergence to equilibrium in the Becker-Döring equations. J. Differential Equations, $191(2): 518-543,2003$.

I. Jeon. Existence of gelling solutions for coagulation- fragmentation equations. Commun. Math. Phys., 567:541-567, 1998.

J. Johansson. Stochastic analysis of nucleation rates. Phys. Rev. E, 93(2):022801, 2016.

F. P. Kelly. Reversibility and Stochastic Networks. Cambridge University Press, Cambridge, 1979.

A. Y. Khinchin. Mathematical Foundations of Quantum Statistics. Translation from the first (1951) Russian ed., edited by I. Shapiro. Graylock Press, Albany, N. Y., 1960.

J. R. King and J. A. D. Wattis. Asymptotic solutions of the Becker-Döring equations with size-dependent rate constants. J. Phys. A, 35(6):1357-1380, 2002.

M. Kreer. Classical Becker-Döring cluster equations: rigorous results on metastability and long-time behaviour. Ann. Physik (8), 2(4):398-417, 1993.

P. Laurençot. Weak solutions to the Lifshitz-Slyozov-Wagner equation. Indiana Univ. Math. J., 50(3):1319-1346, 2001.

P. Laurençot. The discrete coagulation equations with multiple fragmentation. Proc. Edinb. Math. Soc. (2), 45(1):67-82, 2002.

P. Laurençot and S. Mischler. From the Becker-Döring to the Lifshitz-Slyozov-Wagner equations. J. Statist. Phys., 106(5-6): $957-991,2002$

P. Laurençot and D. Wrzosek. The Becker-Döring model with diffusion. I. Basic properties of solutions. Colloq. Math., 75(2): 245-269, 1998

I. M. Lifshitz and V. V. Slyozov. The kinetics of precipitation from supersaturated solid solutions. J. Phys. Chem. Solids, 19(1-2): $35-50,1961$.

B. Linse and S. Linse. Monte carlo simulations of protein amyloid formation reveal origin of sigmoidal aggregation kinetics. Mol. Biosyst., 7:2296-2303, 2011.

A. A. Lushnikov. Coagulation in finite systems. J. Colloid Interf. Sci., 65(2), 1978.

A. H. Marcus. Stochastic coalescence. Technometrics, 10(1):133-143, 1968.

B. Niethammer. On the Evolution of Large Clusters in the Becker-Döring Model. J. Nonlinear Sci., 13(1):115-155, 2003.

B. Niethammer. A scaling limit of the Becker-Döring equations in the regime of small excess density. J. Nonlinear Sci., 14(5): 453-468, 2004

B. Niethammer. Effective theories for Ostwald ripening. In P. Mörters, R. Moser, M. Penrose, H. Schwetlick, and J. Zimmer, editors, Analysis and stochastics of growth processes and interface models, pages 223-243. Oxford Univ. Press, Oxford, 2008.

B. Niethammer and R. L. Pego. On the initial-value problem in the Lifshitz-Slyozov-Wagner theory of Ostwald ripening. SIAM J. Math. Anal., 31(3):467-485, 2000 .

B. Niethammer, F. Otto, and J. J. L. Velázquez. On the effect of correlations, fluctuations and collisions in Ostwald ripening. In A. Mielke, editor, Analysis, modeling and simulation of multiscale problems, pages 501-530. Springer, Berlin, Heidelberg, 2006.

O. Penrose. Metastable states for the Becker-Döring cluster equations. Comm. Math. Phys., 124(4):515-541, 1989.

O. Penrose. The Becker-Döring equations at large times and their connection with the LSW theory of coarsening. J. Statist. Phys., 89(1-2):305-320, 1997. Dedicated to Bernard Jancovici.

O Penrose. The becker-döring equations for the kinetics of phase transitions, 2001.

O. Penrose. Analysis and Stochastics of Growth Processes and Interface Models, chapter Nucleation and droplet growth as a stochastic process, pages 1-12. Oxford University Press, 2008.

O. Penrose and A. Buhagiar. Kinetics of nucleation in a lattice gas model: Microscopic theory and simulation compared. J. Statist. Phys., 30(1):219-241, 1983.

O. Penrose and J. L. Lebowitz. Towards a Rigorous Molecular Theory of Metastability. In E.W. Montroll and J. L. Lebowitz, editors, Fluctuation Phenomena, volume 7 of Studies in Statistical Mechanics, pages 293 - 340. Elsevier, 1979.

O. Penrose, J. L. Lebowitz, J. Marro, M. H. Kalos, and A. Sur. Growth of clusters in a first-order phase transition. J. Statist. Phys., 19(3):243-267, 1978.

S. Prigent, A. Ballesta, F. Charles, N. Lenuzza, P. Gabriel, L. M. Tine, H. Rezaei, and M. Doumic. An efficient kinetic model for assemblies of amyloid fibrils and its application to polyglutamine aggregation. PloS one, 7(11):e43273-e43273, January 2012.

F. Rezakhanlou. Gelation for Marcus-Lushnikov process. Ann. Probab., 41(3):1806-1830, 2013.

H. G. Rotstein. Cluster-size dynamics: A phenomenological model for the interaction between coagulation and fragmentation processes. J. Chem. Phys., 142(22):224101, 2015. 
J. W. P. Schmelzer, editor. Nucleation Theory and Applications. Wiley-VCH Verlag GmbH \& Co. KGaA, Weinheim, 2005.

F. Schweitzler, L. Schimansky-Geier, W. Ebeling, and H. Ulbricht. A stochastic approach to nucleation in finite systems: theory and computer simulations. Physica A, 150:261-279, 1988.

M. Slemrod. Trend to equilibrium in the Becker-Döring cluster equations. Nonlinearity, 2(3):429-443, 1989.

M. Slemrod. The Becker-Döring Equations. In N. Bellomo and M. Pulvirenti, editors, Modeling in Applied Sciences: A Kinetic Theory Approach, Modeling and Simulation in Science, Engineering and Technology, pages 149-171. Birkhäuser Boston, Boston, MA, 2000 .

V. Ssemaganda and G. Warnecke. Existence of metastable solutions for a thermodynamically consistent Becker-Döring model. J. Appl. Anal., 19(1):91-124, 2013.

J. J. L. Velázquez. The Becker-Döring equations and the Lifshitz-Slyozov theory of coarsening. J. Statist. Phys., 92(1-2):195-236, 1998.

J. J. L. Velázquez. On the effect of stochastic fluctuations in the dynamics of the Lifshitz-Slyozov-Wagner model. J. Statist. Phys., 99(1-2):57-113, 2000.

W. Wagner. Explosion phenomena in stochastic coagulation-fragmentation models. Ann. Appl. Proba., 15(3):2081-2112, 2005.

J. A. D. Wattis. An introduction to mathematical models of coagulation-fragmentation processes: a discrete deterministic mean-field approach. Phys. D, 222(1-2):1-20, 2006.

J. A. D. Wattis, C. D. Bolton, and P. V. Coveney. The Becker-Döring equations with exponentially size-dependent rate coefficients. J. Phys. A, 37(8):2895-2912, 2004.

P. Whittle. Statistical processes of aggregation and polymerization. Math. Proc. Cambridge Philos. Soc., 61(2):475-495, 1965.

R. Yvinec, M. R. D’Orsogna, and T. Chou. First passage times in homogeneous nucleation and self-assembly. J. Chem. Phys., 137 (24):244107, 2012.

R. Yvinec, S. Bernard, E. Hingant, and L. Pujo-Menjouet. First passage times in homogeneous nucleation: Dependence on the total number of particles. J. Chem. Phys., 144(34106), 2016.

E. Hingant, Departmento de Matemática, Universidad del Bío-Bío, Concepción, Chile

E-mail address: ehingant@ubiobio.cl

R. Yvinec, Physiologie de la Reproduction et des Comportements, Institut National de la Recherche Agronomique (INRA)

UMR85, CNRS-Université François-Rabelais UMR7247, IFCE, Nouzilly, 37380 France

E-mail address romain.yvinec@tours.inra.fr 